\title{
EVALUACIÓN DEL TRATAMIENTO TÉRMICO DE LAS ARVEJAS ENLATADAS (Pisum sativum) EN SALMUERA
}

\author{
HEAT TREATMENT EVALUATION OF PEAS \\ CANNED (Pisum sativum) IN BRINE
}

\author{
Bibiana Yaneth Gaviria López, ${ }^{1}$ Paola Andrea Mazuera García
}

\section{RESUMEN}

Cuando se habla de los principales requisitos que debe reunir un alimento, sin duda, el más importante es que sea inocuo o seguro para la salud de los consumidores. Es por esto que se hace necesario adoptar más y mejores sistemas o prácticas que permitan garantizar dicha seguridad. La intención de este artículo es describir el diseño de un correcto proceso térmico de vegetales enlatados, para este caso, la arveja y la importancia que tiene su realización. En la parte experimental se realizó el estudio de penetración de calor del producto con el fin de determinar los parámetros de tiempo y temperatura adecuados para obtener un alimento estéril comercialmente. Los datos obtenidos fueron analizados utilizando el método de Ball mediante el software CALSoft 32. Los resultados mostraron que la letalidad objetivo de proceso Fo de 6 fue alcanzada en 17 minutos a una temperatura de $121^{\circ} \mathrm{C}$.

Adicionalmente, se establecieron los procesos alternativos que pueden ser utilizados en caso de desviaciones durante el proceso térmico. Cabe anotar que este trabajo está enfocado a los tratamientos térmicos para envases de hojalata esterilizados en autoclaves verticales de vapor saturado.

Palabras clave: autoclave, esterilización, inocuidad, microorganismo, temperatura, tratamiento térmico.

\begin{abstract}
When we speak about the main requirements that food should fulfill, we know, without a doubt, that it should be innocuous or harmless to the health of consumers. This is why it is becoming more and more necessary to implement systems and practices to ensure this innocuousness. This article is meant to describe the design and the importance of conducting a proper thermal processing of canned vegetables, in this case, green peas. It also aims, to study the heat penetration of the product to be able to determine the appropriate time parameters and temperature that are necessary to obtain a commercially sterile food. The

1 Profesional en Ciencia y Tecnología de Alimentos. Especialización Ingeniería de Procesos de Alimentos y Biomateriales UNAD, Colombia. bibi1106@hotmail.com.

2 Paola Andrea Mazuera, Ingeniera Agroindustrial, Especialista en Gerencia. Especialización Ingeniería de Procesos de Alimentos y Biomateriales UNAD, Colombia. pamazuera@gmail.com.
\end{abstract}


data was analyzed using the Ball method and the CalSoft32 software and the results showed that $F o=6$ (Objective Process Lethality) was achieved in 17 minutes at a temperature of 121 ${ }^{\circ} C$. Additionally, the alternative processes that can be used in case of deviations during the thermal process were established. It's worth mentioning that this work is focused on heat treatments for tin cans which are sterilized in vertical steam saturated autoclaves.

Key words: Heat treatment, safety, sterilization, Microorganism, Autoclave, Temperature

Aprobado: Junio 29 de 2010

\section{INTRODUCCIÓN}

En Colombia, las industrias conserveras suelen diseñar y ejecutar cada uno de sus procesos térmicos con base en referencias bibliográficas o en re aplicaciones de otras industrias, desconociendo la importancia de obtener, bajo métodos científicos, las variables de procesamiento específicas para cada una de ellas, ya que esto va sujeto a las condiciones propias de cada planta, así como a diversos factores, como ubicación geográfica, equipos e instalaciones, productos elaborados y variables críticas, entre otros, incluyendo, como se mencionó anteriormente, el método científico aplicado.

A partir de junio de 2011, entró en vigencia la resolución 2195 de 2010 del Ministerio de Protección Social de Colombia. Dicha resolución establece el "reglamento técnico sobre los requisitos que se deben cumplir durante el proceso térmico de alimentos envasados herméticamente de baja acidez y acidificados que se fabriquen, transporten, expendan, distribuyan, importen, exporten y comercialicen para el consumo humano" (Invima, 2010).

Las industrias conserveras deben garantizar el diseño de los procesos térmicos en la elaboración de sus productos, bajo la utilización de metodologías científicas que garanticen un alimento seguro y confiable, logrado bajo los esquemas microbiológicos particulares para cada uno de ellos.

\section{ANTECEDENTES}

Los enlatados vegetales han ganando una mayor presencia en la canasta familiar, pasando de ser un alimento empleado para ocasiones especiales, a usarse en la alimentación cotidiana. Ofrecen grandes ventajas, entre ellas, la practicidad, conservación durante largos periodos de tiempo, facilidad de preparación y, además, nutrición y seguridad.

En Colombia, la arveja (Pisum Sativum), después del fríjol (Phaseolus Vulgaris), es la leguminosa de más importancia Fenalce (2010), pues se cosecha y comercializa durante todo el año y tiene una de las mayores áreas en hectáreas para su cultivo en comparación con otras hortalizas. El cultivo de la arveja ha sido un factor estabilizador de la economía de los pequeños productores de las zonas y ha contribuido a su seguridad alimentaria. 
Las variedades más comunes que se consumen alrededor del mundo son: la arveja fresca y la arveja seca. Esta última requiere una larga preparación, sujeta al remojo de hasta 12 horas y una prolongada cocción, lo que ha incentivado el consumo de presentaciones industriales pre cocidas, congeladas y enlatadas. Dichas presentaciones deben reunir ciertos requerimientos, entre los cuales, el de mayor relevancia es seguridad para la salud de los consumidores; por tal razón, cada vez se hace más necesario adoptar sistemas o prácticas que permitan garantizar esta seguridad.

Una de las formas más utilizadas para la conservación de los alimentos es la aplicación de calor. En el caso de los alimentos enlatados, dicha conservación se logra mediante la aplicación del proceso térmico de esterilización, con la cual no se requiere la adición de conservantes para garantizar su esterilidad comercial y su vida útil.

En la elaboración de los alimentos enlatados se deben tener evaluados los procesos térmicos de esterilización, lo cual implica determinar el tiempo y temperatura adecuados para asegurar la destrucción de microorganismos bajo metodologías científicas, lo que permitirá a cada empresa contar con el respaldo y la confianza necesarios para procesar en condiciones seguras.

\section{Fundamento del tratamiento térmico}

El proceso térmico de esterilización se determina mediante la aplicación de pruebas de distribución de temperatura en el autoclave y de penetración de calor en el producto que se quiere elaborar. "Se deben establecer factores intrínsecos de este producto como: tipo y resistencia térmica de los microorganismos objetivo, esporas o enzimas presentes en el alimento, pH del alimento, condiciones de calentamiento, propiedades termo físicas del alimento y del envase y condiciones de almacenamiento del producto posteriores al proceso" (Tamayo, 2008).

Las pruebas de distribución de temperatura permiten determinar experimentalmente la operación y comportamiento del autoclave con el fin de garantizar la temperatura de esterilización alrededor de todos los envases por procesar. "Los estudios de penetración de calor se realizan bajo condiciones controladas que representen el peor escenario de producción respecto al tratamiento térmico y que resulten en el modo de calentamiento más lento del producto" (Tamayo, 2008). Se asume que, si el punto con menor razón de calentamiento dentro del envase (punto crítico o punto frío) recibe la cantidad de calor necesaria para alcanzar la esterilidad comercial, entonces, el resto del envase ha recibido la misma o una mayor cantidad de calor y, por consiguiente, todo el producto ha logrado dicha esterilización. Los estudios deben realizarse para cada alimento en particular y es específico para éste, para su formulación, su proceso de elaboración, el tamaño del envase y el tipo de sistema de envase usado. 
Es así como se obtiene la combinación adecuada de tiempo y temperatura para cada referencia, de tal forma que se garantice la esterilidad comercial y la repetibilidad del proceso térmico.

\section{MARCO TEÓRICO}

\section{Proceso térmico de esterilización}

Es la aplicación de calor a un alimento en un envase cerrado herméticamente por un período de tiempo y a una temperatura que ha sido determinada científicamente como adecuada para asegurar la destrucción de los microorganismos que puedan ser perjudiciales para la salud pública.

El conocimiento de la microbiología del alimento y de los métodos de procesamiento es el punto de partida para establecer los procesos térmicos de esterilización en alimentos enlatados. Los microorganismos que deterioran los alimentos están presentes en todos los productos crudos y no pueden crecer bajo condiciones ambientales adversas, ya que mueren si su medio ambiente se torna inadecuado.

Establecer un proceso térmico de esterilización parte del conocimiento de la resistencia térmica de los microorganismos; es decir, de la cantidad de calor requerida para su destrucción y de la velocidad de calentamiento del producto que está siendo esterilizado.

Los procesos de esterilización son llevados a cabo en autoclaves, los cuales son "equipos destinados al tratamiento térmico de un alimento envasado herméticamente cerrado, que trabaja con parámetros de presión y temperatura" (Invima, 2010).

\section{Equipos}

Los autoclaves son de varios tipos: horizontales o verticales y estáticos o discontinuos. Cada proceso que se lleva a cabo en los diferentes equipos utiliza medios de transferencia de calor según su necesidad y diseño; es así como funcionan con agua, mezcla de vapor y aire y, el más eficiente y de bajo costo, vapor saturado. Este último tiene grandes ventajas, ya que alcanza en poco tiempo temperaturas más altas que la ebullición del agua y está soportado en un sistema hermético de presión. 


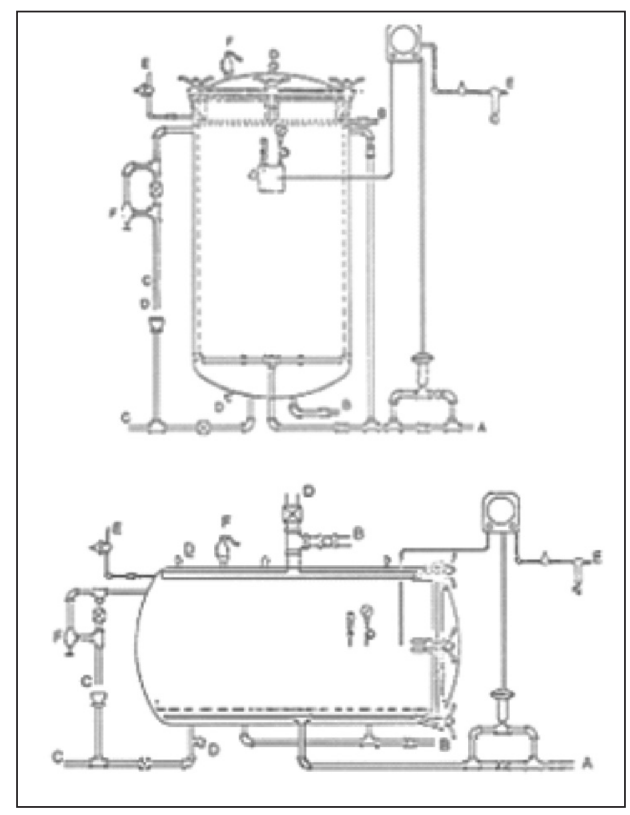

Figura 1. Autoclave estático vertical y horizontal (The Food Process Institute)

\section{Microorganismo indicador}

El microorganismo indicador utilizado con frecuencia en los enlatados es el Clostridium botulinum. Éste es un patógeno anaerobio esporulado productor de una exotoxina (neurotóxica) muy peligrosa para el ser humano que causa botulismo. Sus esporas son resistentes al calor y pueden sobrevivir en aquellos alimentos mínima o inadecuadamente procesados. Este microorganismo no crece a $\mathrm{pH}$ de 4,5 o menor y actividad de agua menor a 0,93 . Para control del Clostridium botulinum, las conservas alimenticias reciben tratamiento térmico drástico, ya que las condiciones presentes dentro del envase sellado son las óptimas para el desarrollo de dicho microorganismo, por lo que se asume su presencia en todos los alimentos poco ácidos previstos a ser enlatados. Por tal razón, su destrucción es el criterio de seguridad alimentaria, utilizado en el diseño de un tratamiento térmico óptimo.

\section{Resistencia térmica de los microorganismos}

La resistencia térmica de los microorganismos depende de varios factores, como son: el tipo de microorganismo, sus características de crecimiento, naturaleza y el tipo de alimento donde se reproducen. Los microorganismos crecen y mueren en forma logarítmica y es teóricamente imposible eliminar por completo el recuento de estos en un alimento. 
El tiempo necesario para reducir el recuento microbiano en un ciclo logarítmico se denomina valor $\mathrm{D}$, el cual depende del tipo de microorganismo y está directamente relacionado con la temperatura. Si se grafican los valores $\mathrm{D}$ de un microorganismo a diferentes temperaturas se obtiene el valor $\mathrm{Z}$, el cual se define como "la constante que representa el cambio de la resistencia del microorganismo respecto a la temperatura" (Qtech, 2008).

Los valores de D y Z para el Clostridum Botulinum son:

$\mathrm{D}\left(121^{\circ} \mathrm{C}\right)=0,21$ minutos y $\mathrm{Z}=10^{\circ} \mathrm{C}$

Los procesos de esterilización comercial buscan reducir 12 veces la contaminación de C. botulinum, es decir 12D.

\section{Letalidad o valor $\mathbf{f}$ de referencia $\mathrm{F}_{0}$}

"La letalidad se define como el tiempo de calentamiento equivalente al efecto durante un minuto a una temperatura de calentamiento referencial establecida generalmente a $121,1^{\circ} \mathrm{C}$ para los procesos de esterilización comercial” (Tamayo, 2008). Es decir, el número de minutos requeridos a una temperatura específica para destruir un número determinado de microorganismos con un valor $\mathrm{Z}$ específico.

Así, para el C. Botulinum, el valor $\mathrm{F}_{0}$ es el número de minutos requeridos para destruir un número específico de microorganismos cuyo valor $\mathrm{Z}$ es de $10^{\circ} \mathrm{C}$ a una temperatura de $121^{\circ} \mathrm{C}$. Con esto, el valor $\mathrm{F}_{0}$ mínimo es $12 \mathrm{D}$, que equivale a 2.52 minutos.

\section{Etapas De Un Proceso Térmico De Esterilización}

El proceso térmico de esterilización se compone de las siguientes etapas:

Etapa de venteo: su objetivo es eliminar el aire contenido en el autoclave, lo que se logra ingresando vapor al equipo y termina cuando todo el aire contenido es removido en su totalidad. Un mal proceso de venteo daría origen a puntos o zonas frías en el autoclave, en las cuales se tendría un procesamiento térmico insuficiente.

Etapa de levante: eliminado el aire en el autoclave, el vapor sigue ingresando hasta alcanzar la temperatura de esterilización. La duración de esta etapa es la necesaria para alcanzar dicha temperatura.

Etapa de sostenimiento (esterilización): alcanzada la temperatura de esterilización, debe ser mantenida sobre los envases hasta que el calor penetre al interior de estos en su punto más frío, logrando, de esta manera, la destrucción de los microorganismos que pueden deteriorar el alimento. Como se mencionó anteriormente, el tiempo requerido para esta etapa depende de la composición del alimento y del tamaño del envase. 
Etapa de enfriamiento: terminada la etapa de esterilización, es necesario llevar lo más rápido posible los envases a temperaturas que permitan su manipulación y empaque. Por lo general, el producto se somete a continuos baños con agua fría que llevan el producto a temperaturas inferiores a $40^{\circ} \mathrm{C}$, con el fin de evitar posible re contaminación en éste.

En la siguiente figura se muestra una curva típica de Temperatura vs Tiempo para un proceso de esterilización en sus diferentes etapas.

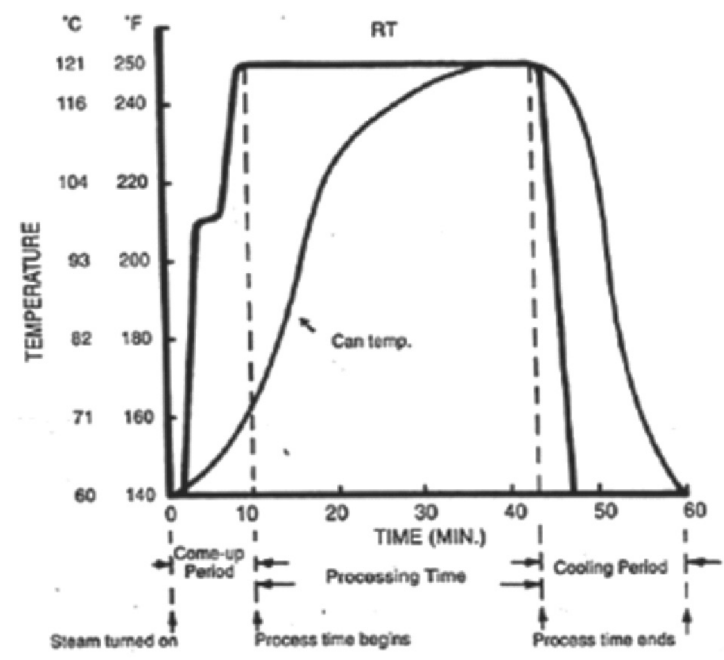

Figura 2. Etapas del proceso térmico de esterilización

Fuente: (A Complete Course in Canning. Volumen 1

\section{Determinación del proceso de esterilización}

Para establecer un proceso térmico de esterilización es necesario encontrar las combinaciones de tiempo y temperatura adecuadas que permitan la repetibilidad del proceso. Para hallar el peor escenario que se pueda dar durante la operación normal de la planta de producción y de esta manera asegurar el proceso térmico de esterilización, se utilizan dos pruebas con objetivos específicos, las cuales son:

Prueba de Distribución de Temperatura (DT): su objetivo consiste en determinar el comportamiento del equipo de procesamiento térmico, conocer su funcionamiento óptimo y saber cómo se distribuye la temperatura al interior del mismo, de manera que sea posible identificar la zona fría dentro del autoclave. Para elaborar esta prueba se colocan termocuplas en cada uno de los canastos en los que se ubican los envases dentro del autoclave, tratando de abarcar la mayor cantidad del volumen del equipo. Una vez puestas las termocuplas, se comienza a ejecutar el proceso de esterilización en condiciones normales de planta, registrando las variables más relevantes del proceso. 
El posterior análisis de la prueba permite determinar la homogeneidad de la temperatura dentro del autoclave, definir el tiempo y temperatura de venteo y el tiempo de levante para alcanzar la temperatura de esterilización deseada.

En la siguiente tabla se observa la dispersión de temperaturas para diferentes puntos en el autoclave, alcanzada durante la ejecución del venteo y la posterior homogenización de los mismos, hasta lograr la temperatura de sostenimiento.

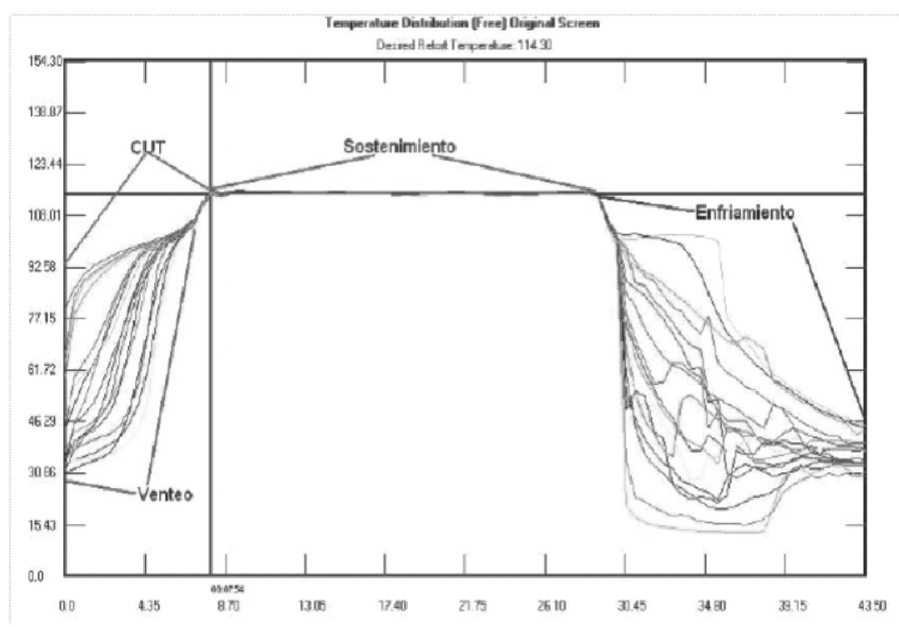

Figura 3. Ejecución de prueba de distribución de temperatura. (QTech, Seminario 2008) Fuente: (QTech, Seminario 2008)

Pruebas de Penetración de Calor (PQ): tienen como objetivo conocer cómo se calienta el alimento durante el proceso térmico de esterilización y determinar, además, la letalidad alcanzada en el punto más frío del envase durante el proceso. Estas pruebas se dividen en dos etapas: la primera es la localización del punto frío, que es el punto que se calienta más lentamente respecto al resto del envase; en la segunda se establece la relación tiempo/ temperatura para alcanzar la letalidad (Fo) deseada.

En la ejecución se introducen termocuplas dentro de cada envase a diferentes alturas, de tal manera que se mantenga el cierre hermético. Los datos obtenidos en el punto más frío (segunda etapa) son graficados y analizados utilizando el método de Ball, mediante el cual se calcula el tiempo y la temperatura requeridos en el proceso de esterilización para garantizar la letalidad esperada en el producto.

El establecimiento del proceso térmico se realiza con los resultados obtenidos durante la correcta aplicación de las pruebas de distribución de temperatura y de penetración de calor. 
A continuación se muestra la ubicación de las termocuplas en los envases para las pruebas de penetración de calor.

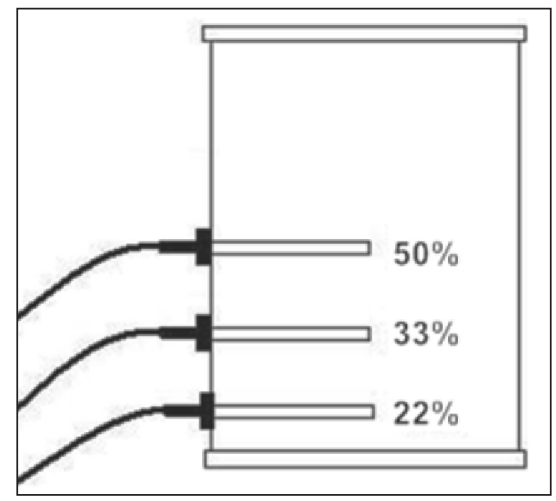

Figura 4. Ubicación de termocuplas en las pruebas de penetración de calor(QTech, Seminario 2008)

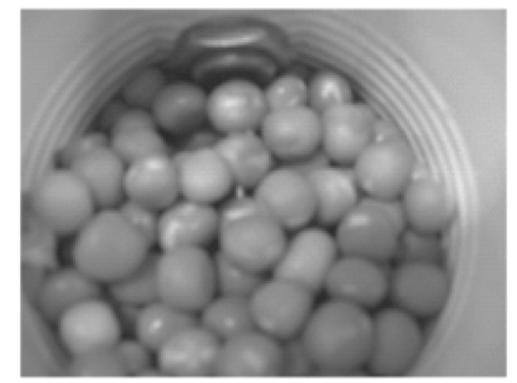

Figura 5. Fotografía del producto con termocupla

\section{Aplicación del proceso térmico a las arvejas en lata}

Antes de detallar las pruebas realizadas en el producto, se describirá el proceso de elaboración de las arvejas en lata:

\section{Proceso de elaboración de la arveja enlatada}

Recepción de materiales: la materia prima utilizada para este proceso es arveja verde deshidratada, la cual se inspecciona al momento de la recepción, para verificar el cumplimiento de las especificaciones establecidas por la empresa.

Limpieza e hidratación de granos: los granos de arveja utilizados en el proceso pasan por un equipo limpiador que retira las impurezas como piedras, palos, entre otros. Luego son sometidos a un proceso de hidratación en tanques con agua, durante un tiempo determinado. 
Selección: la arveja hidratada es seleccionada sobre bandas transportadoras donde se retira material extraño, suciedades y granos defectuosos.

Escaldado: en esta etapa el material se sumerge en agua a temperatura de ebullición con el fin de inactivar las enzimas presentes, disminuir la carga microbiana y fijar los colores del grano.

Elaboración líquido de gobierno: utilizando una marmita abierta con agitador, se prepara el líquido de cobertura o salmuera.

Llenado y sellado: la arveja se introduce en los envases y son cubiertas con el líquido caliente o salmuera. Estos pasan a través de una selladora donde son cerrados herméticamente. En este punto se garantiza el correcto doble cierre y la generación de un espacio de cabeza o espacio libre donde el aire es retirado.

Esterilización y enfriamiento: los envases son sometidos a un proceso térmico a altas temperaturas y presión donde se logrará un producto comercialmente estéril. En esta etapa se logra la destrucción de los microorganismos patógenos.

Etiquetado y empaque: finalmente, las latas son etiquetadas y embaladas en cajas corrugadas para ser distribuidas.

\section{Pruebas Experimentales para Arveja en Lata}

En la fase experimental se procedió a realizar las pruebas de penetración de calor donde se analizaron los factores críticos asociados al proceso y al producto y se realizaron las mediciones bajo las siguientes especificaciones:

Nombre del producto y referencia de envase: "Arvejas en Salmuera". Envase de hojalata tres cuerpos referencia 307 x 409.

Autoclave: vertical de vapor saturado. Envases ordenados (verticales) con uso de separadores.

Equipo: los monitoreos de temperatura se llevaron a cabo con un equipo de adquisición de datos CALPlex (TechniCAL) en conjunto con el programa CALSoft 32, el cual es utilizado para la captura de datos, análisis de estos y cálculo del proceso térmico.

Protocolo de trabajo: se efectuaron dos pruebas de penetración de calor en el producto envasado. Las muestras elaboradas fueron procesadas en vapor saturado. 
Variables del producto: previo a la preparación de las muestras, las arvejas fueron sometidas a un proceso de hidratación durante 12 horas. El grano se lava y selecciona para eliminar impurezas y luego se escalda durante 3 minutos a temperatura de ebullición $\left(90^{\circ} \mathrm{C}\right.$ a $92^{\circ} \mathrm{C}$ ). Aparte, se prepara el líquido de cobertura (agua, azúcar y sal) y posteriormente los envases se llenan con la arveja y la salmuera de $70^{\circ} \mathrm{C}$ a $80^{\circ} \mathrm{C}$.

Las termocuplas se ubicaron en diferentes alturas del envase (al 50\%, 33\% y 22\%) en las cuales se insertaron unidades de arveja.

Factores críticos: para efectos de las pruebas de penetración de calor se utilizó un peso de llenado de $308 \mathrm{~g}$ de arveja (aproximadamente un 10\% superior al máximo utilizado por la empresa) y un peso neto de $600 \mathrm{~g}$.

El calibre de las arvejas fue de $9 \mathrm{~mm}$ de diámetro, que es el que se utiliza normalmente en este producto. Una vez llenos los envases, se hicieron pasar por un túnel de vapor, se sellaron y ubicaron ordenadamente dentro de las canastas de la autoclave.

Variables de proceso: el proceso de esterilización se aplicó siguiendo los procedimientos definidos en los estudios de distribución de temperatura realizados previamente por la empresa. Las condiciones de operación fueron las siguientes:

Venteo mínimo: 6 minutos y hasta, al menos, $108^{\circ} \mathrm{C}$. El drenaje fue cerrado en $95{ }^{\circ} \mathrm{C}$. Operación de venteo con válvula neumática (automática) y uso de válvula “bypass" de vapor.

Condiciones de esterilización: subida de temperatura lo más rápido posible hasta alcanzar la requerida en el proceso. Tiempo mínimo de elevación de nueve minutos, obteniendo un CUT total de 15 minutos, el cual hace referencia al tiempo inicial desde la entrada de vapor hasta alcanzar la temperatura de sostenimiento. La temperatura de proceso fue de $122,5^{\circ} \mathrm{C}$; este valor corresponde a los resultados obtenidos en las pruebas de distribución, donde se encontró que la diferencia entre el punto más frío y caliente del autoclave es de $1,5^{\circ} \mathrm{C}$, lo que garantiza que todos los puntos del equipo estén mínimo a $121^{\circ} \mathrm{C}$.

Variables de las pruebas: los envases con producto se ubicaron en forma ordenada (vertical) en la fila superior de la tercera canasta del autoclave (punto frío). Posteriormente, la canasta con las muestras se completó con envases de hojalata llenos con agua usando separadores entre cada fila de envases. En el autoclave se ubicaron un total de cuatro canastas con envases de hojalata llenos con agua. Durante la ejecución de las pruebas, la planta operó en condiciones de producción normal. 
Se efectuaron dos pruebas de penetración de calor. La primera, correspondió a la determinación de la zona fría al interior del envase. Para ésta, se ubicaron cuatro termocuplas en el centro geométrico: cuatro al $33 \%$ y cuatro al $22 \%$ de la altura total de envase. La zona fría se identificó en dos puntos: al $22 \%$ y al $33 \%$, por lo que en la segunda prueba se colocaron seis termocuplas en cada una de estas posiciones para un total de 12 termocuplas.

Un total de 24 termocuplas válidas se utilizaron en dos ensayos de penetración de calor. En forma previa a las pruebas, el sistema de adquisición de datos se calibró a la temperatura del proceso. Los factores de corrección para cada termocupla (canal) se incorporaron al programa.

Los datos calculados en esta prueba se utilizan para la referencia en estudio (tamaño del envase, peso de llenado y formulación específica). Si se desea cambiar la dimensión de la lata, peso neto del producto o tipo de alimento por esterilizar, se debe validar el proceso para cada condición.

\section{Resumen de Pruebas}

Tabla 1. Resumen de pruebas de evaluación del proceso térmico en arveja enlatada

\begin{tabular}{|c|c|c|c|c|c|c|}
\hline \multicolumn{2}{|r|}{ Prueba } & $\begin{array}{c}\text { Peso } \\
\text { Llenado }\end{array}$ & $\begin{array}{l}\text { Förmula y } \\
\text { preparación }\end{array}$ & $\begin{array}{l}\text { Peso } \\
\text { neto }\end{array}$ & $\begin{array}{c}\text { Posición } \\
\text { envase }\end{array}$ & $\begin{array}{c}\text { Posición } \\
\text { termocuplas }\end{array}$ \\
\hline 1 & $\begin{array}{l}\text { Evaluación } \\
\text { zona fria }\end{array}$ & 308 g máx. & Normal & $600 \mathrm{~g}$ & Vertical & $\begin{array}{l}\text { Centro } \\
\text { geometrico } \\
33 \% \text { altura } \\
22 \% \text { altura }\end{array}$ \\
\hline 2 & Repetición & 308 gmáx. & Nomal & $600 \mathrm{~g}$ & Vertical & $\begin{array}{l}33 \% \text { altura } \\
22 \% \text { altura }\end{array}$ \\
\hline
\end{tabular}

Fuente: los autores.

\section{RESULTADOS}

Los datos Tiempo vs. Temperatura obtenidos en cada monitoreo se graficaron y analizaron utilizando el software CALSoft 32. La evaluación se efectuó empleando el método de la fórmula de Ball y utilizando un valor de letalidad -Fo (121/10)- objetivo de 6.0 minutos. El logro de la esterilidad comercial del producto depende del cumplimiento de los factores críticos definidos en esta prueba.

La siguiente tabla muestra el cuadro completo de procesos alternativos: 
Tabla 2. Cuadro completo de procesos alternativos arrojado por software CALSoft 32

\begin{tabular}{|c|c|c|c|c|}
\hline $\begin{array}{c}\text { l.T. } \\
\text { (deg. C) }\end{array}$ & $\begin{array}{c}R . T . \\
\text { (deg.C) }\end{array}$ & $\begin{array}{l}\text { Calculated } \\
\text { Process Time } \\
\text { (minutes) }\end{array}$ & $\begin{array}{l}\text { Calculated } \\
\text { Process Time } \\
\text { (HH:MM:SS) }\end{array}$ & $\begin{array}{l}\text { Suggested } \\
\text { Process Time } \\
\text { (minutes) }\end{array}$ \\
\hline $\begin{array}{l}30.00 \\
30.00 \\
30.00 \\
30.00 \\
30.00\end{array}$ & $\begin{array}{l}118.00 \\
119.00 \\
120.00 \\
121.00 \\
122.00\end{array}$ & $\begin{array}{l}25.00 \\
22.10 \\
19.65 \\
17.54 \\
15.72\end{array}$ & $\begin{array}{l}00: 25: 00 \\
00: 22: 07 \\
00: 19: 39 \\
00: 17: 33 \\
00: 15: 44\end{array}$ & $\begin{array}{l}25.00 \\
23.00 \\
20.00 \\
18.00 \\
16.00\end{array}$ \\
\hline $\begin{array}{l}40.00 \\
40.00 \\
40.00 \\
40.00 \\
40.00\end{array}$ & $\begin{array}{l}118.00 \\
119.00 \\
120.00 \\
121.00 \\
122.00\end{array}$ & $\begin{array}{l}24.03 \\
21.14 \\
18.70 \\
16.60 \\
14.79\end{array}$ & $\begin{array}{l}00: 24: 02 \\
00: 21: 09 \\
00: 18: 42 \\
00: 16: 37 \\
00: 14: 48\end{array}$ & $\begin{array}{l}25.00 \\
22.00 \\
19.00 \\
17.00 \\
15.00\end{array}$ \\
\hline $\begin{array}{l}50.00 \\
50.00 \\
50.00 \\
50.00 \\
50.00\end{array}$ & $\begin{array}{l}118.00 \\
119.00 \\
120.00 \\
121.00 \\
122.00\end{array}$ & $\begin{array}{l}22.92 \\
20.05 \\
17.62 \\
15.54 \\
13.74\end{array}$ & $\begin{array}{l}00: 22: 56 \\
00: 20: 03 \\
00: 17: 38 \\
00: 15: 33 \\
00: 13: 45\end{array}$ & $\begin{array}{l}23.00 \\
21.00 \\
18.00 \\
16.00 \\
14.00\end{array}$ \\
\hline $\begin{array}{l}60.00 \\
60.00 \\
60.00 \\
60.00 \\
60.00\end{array}$ & $\begin{array}{l}118.00 \\
119.00 \\
120.00 \\
121.00 \\
122.00\end{array}$ & $\begin{array}{l}21.64 \\
18.79 \\
16.37 \\
14.31 \\
12.53\end{array}$ & $\begin{array}{l}00: 21: 39 \\
00: 18: 48 \\
00: 16: 23 \\
00: 14: 19 \\
00: 12: 33\end{array}$ & $\begin{array}{l}22.00 \\
19.00 \\
17.00 \\
15.00 \\
13.00\end{array}$ \\
\hline
\end{tabular}

Fuente: los autores.

\section{ANÁLISIS E INTERPRETACIÓN DE RESULTADOS}

Los resultados obtenidos muestran para cada temperatura inicial del producto (I.T) y la temperatura del proceso de esterilización (R.T) los tiempos calculados y sugeridos para el producto evaluado.

Para el caso específico de la arveja enlatada por $580 \mathrm{~g}$ en las condiciones normales del proceso de producción, la temperatura inicial del producto es de $40{ }^{\circ} \mathrm{C}$ mínimo y la temperatura de proceso es de $121{ }^{\circ} \mathrm{C}$; por lo tanto, el tiempo sugerido por el software para estas dos condiciones es de 17 minutos; cumpliendo con estos parámetros y los factores críticos de la prueba se logra la esterilidad comercial del producto.

Los datos adicionales que se encuentran en la tabla 2 pueden ser utilizados en caso de presentarse alguna desviación en una de las condiciones normales de operación durante el proceso.

A continuación se muestran tres casos:

Si la temperatura inicial se baja de $40^{\circ} \mathrm{C}$ a $30^{\circ} \mathrm{C}$ manteniendo la misma temperatura de esterilización de $121^{\circ} \mathrm{C}$, el tiempo de proceso cambiará de 17 minutos a 18 minutos.

Para el tamaño de envase del producto evaluado: si se incrementa la temperatura inicial del producto $10^{\circ} \mathrm{C}$ más; es decir, a $50^{\circ} \mathrm{C}$, con las mismas condiciones del autoclave, los tiempos de proceso quedarían en 16 minutos. 
Si la temperatura inicial del producto permanece a condiciones normales $\left(40^{\circ} \mathrm{C}\right)$ y la temperatura de operación del autoclave se disminuye en $3^{\circ} \mathrm{C}$, es decir, a $118^{\circ} \mathrm{C}$, los tiempos de esterilización del proceso se incrementan a 25 minutos; 8 minutos más que el proceso normal. Por lo tanto, ésta es una de las variables más críticas que debe controlarse durante el proceso térmico.

\section{CONCLUSIONES}

Las empresas, en cada etapa del proceso de elaboración de un enlatado, deben tener controles para evitar contaminaciones; ya que, mientras más reducida sea la carga microbiana inicial de un producto, se requerirá un menor tiempo de calentamiento para alcanzar la destrucción microbiana requerida. Por lo tanto, un tratamiento térmico adecuado para los enlatados puede ser escaso si la carga microbiana inicial en el producto es muy alta.

En la prueba de penetración de calor efectuada en las arvejas enlatadas, el análisis de letalidad, empleando el método de Ball, arrojó una combinación de tiempo y temperatura de 17 minutos a $121^{\circ} \mathrm{C}$, con lo cual se asegura la esterilidad comercial del producto y la vida útil declarada.

El uso del software CALSoft 32 facilitó la solución numérica de las ecuaciones asociadas al proceso térmico ofreciendo un proceso seguro. De igual forma, muestra alternativas que pueden usarse para corregir desviaciones durante la esterilización.

Durante las pruebas se logró identificar que la variable más crítica por controlar durante el proceso térmico es la temperatura de esterilización (T.R), ya que, una desviación en este parámetro afecta significativamente los tiempos. De no ser corregida oportunamente esta desviación, el producto no cumpliría con la esterilidad comercial, ocasionando pérdidas económicas para la empresa y dejando en riesgo la salud de los consumidores.

\section{REFERENCIAS BIBLIOGRÁFICAS}

CASTRO, M. 2008. Cálculo de Procesos Térmicos de Alimentos en el siglo XXI. Tomado de:http://www.captura.uchile.cl/jspui/bitstream/2250/5171/1/Paper2CastroE.pdf. Consulta realizada en Febrero de 2011.

DOWNING, D. 1996. A Complete Course in Canning. Volumen 1, Fundamental information on canning. Woodhead Publishing Limited. New York.

DOWNING, D. 1996. A Complete Course in Canning. Volumen 2, Microbiology, packaging, HACCP and ingredients. Woodhead Publishing Limited. New York.

DOWNING, D. (1996). A Complete Course in Canning. Volumen 3, Processing procedures for canned food products. Woodhead Publishing Limited. New York. 
EVOLUCIÓN DEL CONSUMIDOR COLOMBIANO - Caso alimentos enlatados 2010. Tomado de:http://ocarmonav.blogspot.com/2010/03/evolucion-del-consumidorcolombiano.html. Consulta realizada en Abril de 2010.

FENALCE. 2010. El cultivo de la arveja, historia e importancia. Tomado de: http://www. fenalce.org/arch_public/arveja93.pdf. Consulta realizada en Abril de 2011.

GUÍA DE BUENAS PRÁCTICAS PARA LA ELABORACIÓN DE CONSERVAS VEGETALES. (s.f.). Tomado de: http://www.rlc.fao.org/es/agricultura/bpa/normtec/ varios/37.pdf. Consulta realizada en Abril de 2010.

MÓDULO DE INVESTIGACIÓN EN IPAB (s.f.). 2005. Especialización en Ingeniería de Procesos en Alimentos y Biomateriales.

PRODENVASES CROWN S.A. 2005 .Tratamientos Térmicos de Conservas en hojalata.

QTECH. 2008. Validación de Procesos térmicos. Diseño y ejecución de pruebas

QTECH. 2008. Validación de Procesos térmicos. Análisis e interpretación de resultados.

RESOLUCIÓN 2195 de 2010.Invima. Tomadode:http://web.invima.gov.co/portal/documents/ portal/documents/root/normatividad/alimentos/RESOLUCION202195/20DE/20/2010. pdf. Consulta realizada en marzo de 2011.

TAMAYO, J. 2008. Diseño y optimización del tratamiento térmico para sopa de fréjoles enlatada en envases de formato A6. Escuela Superior Politécnica del Litoral. En http:// www.dspace.espol.edu.ec/bitstream/123456789/9044/1/D-39894.pdf. Consulta realizada en febrero de 2010.

TAMAYO, M. 2003. El proceso de la investigación científica. México.

THE FOOD PROCESS INSTITUTE. 1982. Alimentos enlatados principio de control del procesamiento térmico, acidificación y evaluación del cierre de los envases. Washington.

VÁSQUEZ, J., SÁNCHEZ, M. 2010. Estudio de Penetración de Calor en una Conserva de Camarón Envasada en Empaque Flexible. En http://www.dspace.espol.edu.ec/ bitstream/123456789/2401/1/4741.pdf. Consulta realizada en Marzo de 2010. 